\title{
Flujo espiratorio en músicos de viento, aplicación al razonamiento de las distancias frente al COVID-19.
}

\author{
Sergio Soto Soria ${ }^{1}$, Jaume Rosello Castello ${ }^{2}$, Ana Valero García ${ }^{3}$, Alberto Caballero \\ García ${ }^{4}$ y Alfredo Córdova Martíne: ${ }^{1}$ \\ ${ }^{1}$ Dpto. Bioquímica, Biología Molecular y Fisiología. Facultad de Ciencias de la Salud. \\ Universidad Valladolid. Campus Universitario "Los Pajaritos". Soria, España. \\ ${ }^{2}$ Conservatorio de Música de Jávea. Jávea, Alicante, España. \\ ${ }^{3}$ Conservatorio "Vicente Perelló" de Benimelli. Benimelli, Alicante, España. \\ ${ }^{4}$ Dpto. de Anatomía y Radiología. Facultad de Ciencias de la Salud. Universidad \\ Valladolid. Campus Universitario "Los Pajaritos". Soria, España.
}

Palabras clave: espirometría; músicos de viento; distancia de seguridad; COVID-19.

Resumen. Los músicos que tocan instrumentos de viento necesitan una función pulmonar efectiva y una fuerza muscular espiratoria adecuada para generar sonido por la vibración de la columna de aire que hay en su interior. La medida de volúmenes y capacidades constituye un importante escalón en las pruebas de función pulmonar. El objetivo de este estudio es evaluar los flujos espiratorios en músicos de viento y valorar si con esos datos se puedan extraer conclusiones acerca de la distancia de seguridad que actualmente están recomendando las autoridades sanitarias para protección frente al COVID-19. El estudio se realizó en músicos de la banda municipal de Soria. En 27 músicos y 19 sujetos controles, de edades entre 19 y 29 años, se analizaron la capacidad vital forzada (CVF), el volumen espiratorio forzado en 1 segundo (FEV1), el flujo espiratorio máximo (PEF) y el índice de Tiffeneau (FEV1/CVF). Observamos que la CVF, el FEV1y el PEF valores menores en el grupo de los músicos. El índice de Tiffeneau (la relación FEV1/CVF), en ambos grupos se mantuvo dentro de los rangoos fisiolóǵicos de la normalidad, sin diferencias significativas. Creemos que en el caso de los músicos debería ser reconsiderada la distancia de seguridad establecida actualmente como prevención de contaǵio del COVID-19, puesto que los flujos espiratorios son menores. Además, se debe considerar el volumen de aire que se queda en los tubos del instrumento y que constituye el "espacio muerto". Es decir, en el músico, además de ejercer menor presión para el flujo respiratorio, este se controla mucho más.

Autor de correspondencia: Alfredo Córdova Martínez. Dpto. de Anatomía y Radiología. Facultad de Ciencias de la Salud. Universidad Valladolid. Campus Universitario “Los Pajaritos”. Soria, España. Correo electrónico: a.cordova@ uva.es 


\title{
Expiratory flow in wind musicians, application to the consideration of distances against COVID-19.
}

\author{
Invest Clin 2021; 62 (1): 16-27
}

Key words: spirometry; wind musicians; safety distance; COVID-19.

\begin{abstract}
Musicians who play wind instruments need effective lung function and adequate expiratory muscle strength to generate sound due to the vibration of the air column in their interior. The measurement of volumes and capacities constitutes an important step in pulmonary function tests. The objective of this study was to evaluate the expiratory flows in wind musicians, and to assess whether, with these data, conclusions can be drawn about the safety distance that health authorities are currently recommending for protection against COVID-19. The study was carried out in 27 musicians from the Soria's (Spain) municipal band, and 19 controls, aged between 19 and 29 years. The forced vital capacity (FVC), forced expiratory volume in one second (FEV1), maximum expiratory flow (PEF) and the Tiffeneau index (FEV1/CVF) were analyzed. We observed that the CVF, FEV1 and PEF were lower in the group of musicians. The Tiffeneau index (the FEV1 / CVF ratio) in both groups remained within the physiological ranges of normality, without significant differences. We think that in the case of musicians, the safety distance currently established to prevent the spread of COVID-19 should be reconsidered, since expiratory flows are lower. In addition, the volume of air that remains in the instrument tubes and that constitutes the "dead space" must be considered. That is, in the musician, in addition to exerting less pressure for the respiratory flow, this is much more controlled.
\end{abstract}

Received: 15-10-2020 Accepted: 13-12-2020

\section{INTRODUCGIÓN}

El intercambio de gases pulmonares exige que el aire sea movilizado periódicamente mediante la inspiración y la espiración. El volumen de gas que ocupa los pulmones depende de la distensibilidad pulmonar entre los pulmones y caja torácica a lo largo del ciclo respiratorio (1). La medida de volúmenes y capacidades constituye un importante escalón en las pruebas de función pulmonar ya permiten la evaluación de un paciente sospechoso de presentar una enfermedad respiratoria (2).
El coronavirus de tipo 2 causante del síndrome respiratorio agudo severo 2 abreviado (SARS-CoV-2), es un tipo de coronavirus causante de la enfermedad por coronavirus de 2019 (COVID-19). La transmisión de la COVID-19, aunque no está bien caracterizada, parece ser que uno de los mecanismos importantes de propagación es por microgotas (gotitas) y rutas aéreas (3). Se cree que la propagación en el aire ocurre cuando las partículas respiratorias de menos de $5 \mu \mathrm{m}$ de diámetro son inhaladas. La Organización Mundial de la Salud (OMS) informa que puede ocurrir una transmisión en el aire, pero

Vol. 62(1): 16 - 27, 2021 
solo cuando se realizan procedimientos de generación de aerosol. La transmisión por gotículas es distinta de la transmisión aérea, pues esta última tiene lugar a través de núcleos goticulares que contienen microbios. Los núcleos goticulares, que tienen un diámetro inferior a $5 \mu \mathrm{m}$, pueden permanecer en el aire durante periodos prolongados y llegar a personas que se encuentren a más de un metro de distancia (4). La dispersión de las gotas depende de varios factores, como la viscoelasticidad del fluido de espiración, el tipo de ventilación, la velocidad de espiración, la tasa de evaporación y la dinámica de la nube turbulenta generada durante las exhalaciones, los estornudos o la tos (5). Los estornudos y la tos pueden formar una nube de gas multifásico turbulento que puede extender la propagación de una gota y permitirle viajar más lejos. Tras esto, la nube pierde impulso y las gotas se evaporan formando núcleos de gotas que permanecen suspendidos durante horas, con la capacidad de causar una transmisión infecciosa de mayor alcance (5-7).

Los gases presentan una gran compresibilidad, que influye sobre las características del flujo, ya que tanto el volumen como la densidad varían con facilidad. En el caso de los gases el movimiento térmico vence a las fuerzas atractivas $\mathrm{y}$, por tanto, tienden a ocupar todo el volumen del recipiente que los contiene. En los gases, la densidad depende de la presión y de la temperatura.

La comprensión de la evaporación y dispersión de las gotitas y los núcleos de las gotitas es importante para desarrollar métodos efectivos de control para las enfermedades transmisibles por vía aérea. La distancia a la que se pueden transmitir las enfermedades, está condicionada por la distancia la que se pueden propagar las gotículas $(5,6)$. Una vez que se emiten las gotas al ambiente los factores principales que determinan cómo se mueven son su tamaño y las corrientes de aire.

Algunos estudios $(8,9)$ acerca de la transmisión horizontal de gotas muestran distancias de más de 2 metros. Además, existen otros factores que podrían actuar en la transmisión como son gotas respiratorias grandes, objetos y superficies contaminadas y las mencionadas microgotas respiratorias o aerosoles. El número de partículas se reduce con una distancia creciente desde la fuente. Las partículas más grandes generalmente toman una trayectoria balística, es decir, la propia inercia y a las fuerzas inherentes al medio en el que se desplaza (principalmente la fuerza gravitatoria), recorren distancias más cortas. Dependiendo de la densidad de la gota, el diámetro aerodinámico y el impulso, las gotas pueden moverse más rápido, más lento o a la misma velocidad que la corriente de aire con la que se exhalan $(5-7,10)$. El núcleo de la gota cambia con el tiempo, dependiendo de las condiciones medioambientales. La humedad en el aire altera la tasa de evaporación y por ende, su tamaño. Las gotas en un aire seco se evaporan rápidamente reduciendo su tamaño y caen por gravedad más lentamente. Este cambio en el tamaño afecta la manera en que la partícula responde a los patrones de flujo del aire y su asentamiento (11).

Morawska y Cao (12) indican que es muy importante reconocer que el virus se propaga por el aire y recomienden que se implementen medidas de control adecuadas para evitar su mayor propagación, en particular la eliminación de las gotas cargadas de virus en aire interior, por ventilación.

Las mediciones del flujo de aire y el volumen pulmonar se utilizan para realizar la valoración funcional pulmonar (1). La espirometría forzada incluye la medición FEV1 y la CVF, indicadores del estado de la vía aérea. El flujo espiratorio máximo (PEF) es un parámetro dependiente del esfuerzo coordinado voluntario, de la fuerza muscular, del calibre de las vías aéreas, del volumen pulmonar y de las características viscoelásticas del pulmón. La relación FEV1/CV (Índice de Fiffeneau) $<70 \%$ es el parámetro más importante para la identificación de una obstrucción $(1,13)$. 
Los músicos que tocan instrumentos de viento necesitan una función pulmonar efectiva y una fuerza muscular espiratoria adecuada para generar sonido al mover la columna de aire en cada instrumento (14). Hay que tener en cuenta que los músicos son particularmente propensos a los esfuerzos respiratorios excesivos, y un rendimiento prolongado de los instrumentos de viento puede conducir a la sobrecarǵa de los músculos respiratorios (15). Además, producir altas presiones en la boca (zona de embocadura) puede conducir a problemas oclusales (dentales y labiales) y velocidades muy elevadas de aire exhalado e inhalado (16).

Dependiendo de la actividad metabólica, la ventilación tiene que adaptarse aumentando el aire corriente para realizar así una respiración económica. Ello condiciona las tasas del flujo respiratorio, elevándose a medida que se incrementan las necesidades metabólicas y el requerimiento físico, movilizando un volumen de aire muy alto (17). Estas circunstancias en los momentos actuales de la pandemia por la COVID-19 pueden determinar el comportamiento y las medidas de seguridad que se debieran tomar a la hora de desarrollar su profesión como músicos (y en particular, como músicos de instrumentos de viento).

Este estudio se llevó acabo en los meses de noviembre, diciembre (2019), enero y febrero (2020), como cuerpo fundamental de un estudio de Tesis Doctoral, que, aunque más amplio, una parte es dedicada a la función respiratoria. Creemos que puede ser de interés científico conocer la respuesta pulmonar de músicos frente a sujetos de control, en una situación como la actual pandemia. En este estudio nos propusimos evaluar la eficiencia del sistema respiratorio (parámetros de espirometría dinámica) en músicos de viento y analizar los flujos respiratorios, con el fin de poder realizar una mejor determinación de la distancia de seguridad frente al contagio por la COVID-19. Específicamente, pretendemos aportar algunos elementos de discusión que permitan extraer conclusiones a tener en cuenta respecto a la distancia de seguridad que actualmente están recomendando las autoridades sanitarias.

\section{METODOLOGÍA}

En el estudio participaron dos grupos de sujetos, todos ellos varones (Tabla I): a) 27 sujetos músicos (grupo A) y b) 19 sujetos controles (Grupo B). Este segundo grupo (controles) se trata de individuos voluntarios jóvenes universitarios, estudiantes de ciencias de la salud que no tocaban instrumentos de viento. Todos los músicos eran titulados en el "Conservatorio de Música de Soria”, grado profesional, con experiencia musical profesional de más de 10 años. Para conocer la experiencia musical y las horas dedicadas a la música se les preguntó acerca del instrumento que toca, años de experiencia, horas que toca al día, horas que toca por semana. Los instrumentos que tocaban eran al 50\%, viento-metal/viento-madera.

TABLA I

CARACTERISTICAS ANTROPOMÉTRICAS DE LOS GRUPOS.

\begin{tabular}{lcc}
\hline & Grupo A (músicos) & Grupo B (control) \\
\hline $\mathrm{n}$ & 27 & 19 \\
Edad (años) & $29,16 \pm 7,2$ & $19,36 \pm 2,3$ \\
Peso (Kg) & $84,4 \pm 15,3$ & $74,69 \pm 14,5$ \\
Estatura (cm) & $171,7 \pm 14,1$ & $177,0 \pm 9,5$ \\
IMC $\left(\mathrm{Kg} / \mathrm{m}^{2}\right)$ & $28,9 \pm 6,2$ & $23,86 \pm 3,9$ \\
Años de experiencia musical & $18,0 \pm 2,1$ & \\
Tiempo de entreno semanal (h/semana) & 19 & \\
\hline
\end{tabular}

Datos expresados como media \pm desviación estándar $(\mathrm{X} \pm \mathrm{SD})$.

Vol. 62(1): 16 - 27, 2021 


\section{TABLA II}

VALORES ESPIROMÉTRICOS DE LOS SUJETOS EN AMBOS GRUPOS: MÚSICOS (GRUPO A) Y CONTROLES UNIVERSITARIOS (GRUPOS B).

\begin{tabular}{lccc} 
& INDICA & Grupo A & Grupo B \\
& MÚSICOS & $5,28 \pm 0,9^{*}$ \\
FVC (L) & Volumen & $4,61 \pm 0,68$ & $4,66 \pm 0,75^{*}$ \\
FEV1 (L/seg) & Flujo & $4,16 \pm 0,68$ & $88,59 \pm 5,16$ \\
FEV1/FVG (\%) & Valor absoluto & $87,62 \pm 8,1$ & $608,0 \pm 48,56^{*}$ \\
PEF (L/min) & Flujo & $588,73 \pm 59,85$ & \\
\hline
\end{tabular}

Datos expresados como media \pm desviación estándar $(\mathrm{X} \pm \mathrm{SD})$. Diferencia estadística * $\mathrm{p}<0,05)$.

Ninguno de los participantes era fumador o tenía o había tenido enfermedades crónicas tipo alérgiicas o infecciones virales, ni cardíacas, que pudieran ser causa de exclusión del estudio. También fueron excluídos aquellas personas (músicos o no) que tuvieran problemas de escoliosis, pues ello podría condicionar el resultado de los datos espirométricos. Previamente a comenzar la realización de las pruebas, todos los sujetos fueron informados detalladamente sobre el objetivo del estudio y sus procedimientos, pudiendo plantear cuantas preguntas requerían, tras lo cual firmaron el correspondiente permiso informado.

A todos ellos se les realizó prueba espirométrica con el uso de un espirómetro calibrado modelo: MINISPIR LIGHT marca: Medical International Research (Roma-Italia) para obtener los parámetros espirométricos básicos utilizados y analizados en este estudio. El propio aparato nos indica los valores predictivos que tiene establecidos para cada uno de los parámetros analizados, en función con las características antropométricas y demográficas de los sujetos de prueba, lo que permite identificar posibles trastornos respiratorios y sus tipos.

Las valoraciones se realizaron en condiciones constantes: sujeto sentado, con las piernas dobladas a un ángulo de $90^{\circ}$, los pies en el suelo. No utilizaban ropas compresivas. El espirómetro se conectaba al sujeto mediante una boquilla insertada firmemente en la boca del sujeto y se aplicó una pinza oclusora en la nariz. Se realizaron tres manio- bras con una variabilidad inferior al 5\% para FEV1 y CVF, seleccionándose la mejor curva. En las maniobras de respiración se les indicó realizar una inspiración máxima, rápida hasta la posición de la capacidad pulmonar total (TLC), seguida de una espiración rápida y continuada hasta desalojar el máximo volumen pulmonar posible.

Se analizaron los siguientes parámetros espirométricos:

- Capacidad vital forzada (CVF): cantidad de aire que se moviliza en una inspiración o espiración máximas forzadas.

- Volumen espiratorio forzado en 1 segundo (FEV1): cantidad de aire que se moviliza en el primer segundo de una espiración forzada. El FEV1 es el parámetro más empleado de función pulmonar. Es dependiente del esfuerzo, tiene una elevada reproducibilidad y se correlaciona lineal e inversamente con la obstrucción de las vías aéreas.

- Flujo espiratorio máximo (PEF)(L/ min): y sería el que puede exhalarse por segundo en una espiración forzada. A diferencia del FEV1, el PEF se produce más precozmente, se consigue al haber espirado el 75-80\% de la capacidad pulmonar total dentro de los primeros $100 \mathrm{~ms}$ de espiración forzada (alrededor de 0.125 segundo). EL PEF es un parámetro dependiente del esfuerzo, de la fuerza muscular del diafragma, del calibre de las vías aéreas, del volumen pulmonar y de las características vis- 
coelásticas del pulmón. Es decir, representa una medida sencilla, cuantitativa y reproducible de la obstrucción de las vías aéreas.

- Índice de Tiffeneau (FEV1/CVF en \%). El valor normal de la relación es del $80 \%$, y cifras menores son el indicador espirométrico más sensible de obstrucción bronquial al flujo aéreo.

Como es conocido, los valores espirométricos pueden cambiar para una misma persona a lo largo del día, la semana y del año, por lo que todas las pruebas se realizaron a la misma hora del día, siendo en el periodo de 5-6 de la tarde, dada la actividad estudiantil de los sujetos estudiados, tanto músicos, como universitarios.

Los resultados fueron tratados estadísticamente mediante el paquete de software SPSS 12.1. Para describir una distribución normal se utilizó la prueba de KołmogorowSmirnov, y para analizar las diferencias entre grupos con distribución normal se utilizó la prueba t de Student para muestras independientes. Como nivel estadísticamente significativo, $\mathrm{p}<0,05$ fue adoptado.

El estudio fue avalado por el comité de ética de investigación clínica acreditado del área de Salud de Burgos y Soria (Ref. CEIC 1984). Los resultados presentados en este estudio forman parte de un proyecto científico más amplio con el objeto de estudio para la realización de Tesis Doctoral.

\section{RESULTADOS}

Con excepción de la edad, las características de los grupos fueron similares, así que no se generan variaciones en los resultados espirométricos debidas a diferencias en los grupos muestrales (Tabla I). Hay que remarcar que la experiencia de los músicos (grupo A) tenían en promedio, más de 10 años de experiencia, y entrenaban 20 horas por semana. Los músicos eran semiprofesionales integrantes de la banda municipal de Soria.
En la Tabla II se presenta los resultados correspondientes a los datos espirométricos de ambos grupos. Como podemos observar, la CVF era menor en el grupo de los músicos (grupo A) que en el de los controles universitarios (grupo B).

Igualmente, al analizar la FEV1, vimos que fue también menor en el grupo de los músicos (grupo A) con respecto al grupo $\mathrm{B}$ de universitarios no músicos.

Por su parte, el índice de Tiffeneau (la relación FEV1/CVF), en ambos grupos se mantuvo dentro de los rangos fisiológicos de la normalidad, sin diferencias significativas.

En cuanto a los valores de PEF, observamos valores más bajos en los músicos (grupo A) con respecto al grupo de control.

\section{DISCUSIÓN}

Los músicos de instrumentos de viento dedican mucho tiempo a ensayar, lo que representa poner a prueba el sistema respiratorio debido al esfuerzo que ello requiere. Pero estas actividades no solo se realizan en solitario durante el entrenamiento, también requieren entrenamientos grupales para poder ensamblar y perfeccionar las piezas musicales que se interpretan posteriormente ante el público. Tanto durante los ensayos como durante las interpretaciones (conciertos), estos profesionales deben tener una función y fuerza pulmonar adecuada para producir el sonido musical en el instrumento. Este movimiento aéreo durante su respiración implica a su vez la dispersión en el aire de gotitas (elemento transmisor del coronavirus).

En nuestro estudio, limitado a un grupo pequeño de músicos varones, hemos observado una disminución de la capacidad vital (CV) acompañada de valores fisiológicos normales del índice de Tiffeneau (FEV1/ FVC\%) (por encima del 87\%). Además, hemos observado valores de función pulmonar referentes al FEV1 más bajos que el grupo de control. Nuestros datos comparados con los valores resultantes de los estándares de Baldwin, Berglund, Kory, Knudson, Morris,

Vol. 62(1): 16 - 27, 2021 
Nikodemowicz, CECA son considerados normales (18). Bien es verdad que son significativamente menores, pero al estar en los rangos de la normalidad no supone ninguna limitación para el desarrollo de su profesión musical.

Rohwer (19) analizó el comportamiento de la función respiratoria antes y después de un ensayo con su banda de música y no encontró cambios significativos. Esto puede deberse al hecho de que las técnicas de interpretación, como las respiraciones escalonadas y la respiración instantánea, ayudan a los músicos a soplar. Como explica Hajda (20) en su método de clarinete la respiración debe ser uniforme y lenta. Se recomienda combinar la respiración del estómagoo (diafragmática) y las costillas según el diafragma. Este fenómeno que para los músicos es lógico, viene sustentado en el fenómeno fisiológico del punto de igual presión (PIP), que ejerce como factor limitante durante la inspiración (1). Parece que los instrumentistas de viento no mostraron ni un volumen corriente alto (VC) ni una capacidad vital (CVF) alta a pesar de lo que pudiéramos presuponer.

Sin embargo, este fenómeno podría ser aplicado al resto de los instrumentos de viento, es decir, mantener el flujo respiratorio sin hacer esfuerzos extraordinarios, lo que hace que sea un flujo respiratorio menor y regulado que cuando lo haría una persona normal. Es decir, una aplicación efectiva del fenómeno del PIP anteriormente descrito (1). Pero, a pesar del PIP habría que tener en cuenta la diferencia de tocar fuerte o piano pues el esfuerzo y el flujo de salida de la boca es diferente, aunque a la hora de la expulsión del aire a través del instrumento quede atenuada debido al espacio muerto existente a lo largo del tubo. En este sentido, tampoco Borgia y col. (21) y Nauratil y Rejsek (22) encontraron diferencias significativas en los músicos de instrumentos de viento en comparación con otras personas.

Referente al PEF y FEV, Zuskin y col. (23) indicaron que los músicos de instru- mentos de viento tenían un FEV1 significativamente mayor que los sujetos controles, y aquellos intérpretes con más tiempo de empleo tuvieron los mayores aumentos en la función pulmonar. Sin embargo, en nuestro estudio observamos que los niveles de FEV1 eran menores en los músicos que en los controles. De igual forma ocurrió con el PEF. Desde nuestro punto de vista esto es un fenómeno fisiológico normal, pues la educación musical para tocar los instrumentos de viento se basa en ser económicos y regular el flujo respiratorio. Quizás por ello los músicos pudieron llevar a cabo espiraciones más largáas y controladas, mientras que el grupo de control, realizaba espiraciones más cortas y explosivas. Como ya hemos mencionado anteriormente Hajda (20) ya indica la importancia del mantenimiento del flujo respiratorio con el mínimo esfuerzo, hecho avalado por el fenómeno del PIP, que hace que solo una parte de la exhalación de aire sea dependiente del esfuerzo.

En términos de patología podríamos decir que el tiempo forzado de larga duración en las vías respiratorias podría afectar el sistema respiratorio, sin embargo, en nuestro estudio no hemos observado alteración alguna. Zuskin y col. (23) sugieren que por el hecho de observar alto valor de PEF\% y FEV1\%, podría ser así. Sin embargoo, no hemos encontrado estudios que corroboren esta situación. Por el contrario, en nuestro estudio observamos que tanto la FEV1como el PEF son menores en los músicos que en los sujetos control.

Además, hay estudios $(24,25)$ que indican que los músicos de instrumentos de madera se adaptan mejor a volúmenes respiratorios más bajos, sin embargó, la trompeta por ejemplo requiere presiones muy altas, pero bajos caudales de aire. Cossette y col. (26) concluyen que, en los trompetistas jóvenes, las presiones respiratorias máximas son más altas que en los jóvenes que no tocan instrumentos de viento e indican que esto es probablemente una consecuencia del entrenamiento muscular respiratorio con un 
instrumento de viento. Parece ser, al menos teóricamente, que es importante considerar las diferencias entre los instrumentos, ya que unos requieren presiones mayores y por lo tanto requieran mayores demandas respiratorias. Sin embargo, Fuhrmann y col. (27) no encontraron diferencias significativas en los parámetros respiratorios (basadas en los perfiles de presión) de músicos de viento.

La mayoría de los estudios a sugieren que el aumento de los flujos respiratorios y del volumen corriente se consideran un activo para tocar instrumentos de viento $(28,29)$. Según estos autores, tener mayores volúmenes de aire a su disposición le da al músico mayor 'poder' con el cual crear, manipular y mantener el tono musical en un instrumento. Pero es probable que la capacidad de los músicos para controlar el flujo de aire esté más relacionada con su habilidad que con su capacidad física pulmonar.

Por otra parte, y aunque es cierto que las cifras del flujo respiratorio aumentan cuando se incrementan las necesidades metabólicas y el requerimiento físico, lo que conlleva mayor volumen de aire movilizado, en el caso de los músicos es un hecho a reconsiderar. Como ya hemos discutido, el músico al no precisar de un flujo inmediato tan elevado, en las circunstancias de contaminación por la COVID-19 genere menos aerosoles lo que llevaría a replantear las medidas de seguridad que se debieran tomar en el ámbito de los músicos que tocan instrumentos de viento. Sin embargo, ello no implica que tenga menos probabilidades de propagar la COVID-19.

La dispersión de las gotas depende de varios factores, como la viscoelasticidad del fluido de espiración, el tipo de ventilación, la velocidad de espiración, la tasa de evaporación y la dinámica de la nube turbulenta generada durante las exhalaciones, los estornudos o la tos $(5,6)$. Así pues, la comprensión de la evaporación y dispersión de las gotitas y los núcleos de las gotitas es importante para desarrollar métodos efectivos de control para las enfermedades infecciosas.
La distancia a la que se pueden mover las gotas está relacionada con la distancia a la que se pueden transmitir las enfermedades transmitidas por las gotas $(5,6)$. Según recoge la OMS, estar a menos de un metro de distancia de una persona con síntomas respiratorios, como tos o estornudos, sí supone un riesgo de contagío. A esa distancia, estamos expuestos a las gotitas respiratorias con capacidad infectiva, de entre 5 y 10 micrómetros (un cabello humano tiene más de 100 micrómetros de diámetro). Estos aerosoles contienen partículas virales viables que permanece flotando el en aire durante horas. La Organización Internacional de Trabajo (OIT) ha recomendado una distancia mínima de 2 metros entre trabajadores para prevenir los contagios, siempre que se encuentren en sitios bien ventilados $(4,29)$. Por tanto, una persona ubicada cerca durante ese breve periodo de tiempo se puede infectar (31).

Por lo que respecta a la transmisión por el aire, especifica el informe de la OMS, esta se refiere a partículas extremadamente pequeñas, de menos de 5 micrómetros de diámetro, que suelen resultar de la evaporación de gotas más grandes. Se denominan aerosoles y sí que pueden estar en el aire suspendidas durante periodos más largo de tiempo, en función del calor y la humedad, y transmitirse a distancias mayores de un metro. No obstante, señala la OMS, en el caso del SARS-CoV-2 sólo lo hacen en circunstancias muy específicas como las que se dan en el entorno hospitalario, donde el personal sanitario tiene que realizar procedimientos como intubar al paciente, desconectarlo de la ventilación, o hacerle una traqueotomía, que pueden generar esos aerosoles.

Por ello, es oportuno revisar, al menos en el ámbito de la música, la reǵla de control de infección de 1 a $2 \mathrm{~m}$, que establece pautas para las precauciones de gotas.

$\mathrm{Si}$ a ello añadimos que el aire no sale solo por un orificio final (la campana) sino también por las llaves, por lo que la fuerza del flujo de aire exhalado queda muy reducida. En este sentido, hemos de recordar que,

Vol. 62(1): 16 - 27, 2021 
por ejemplo, en condiciones de flujo estable (técnica que realiza el músico habitualmente para administrar el aire durante mayor número de compases, el aire fluye en el tubo a modo de capas concéntricas de iǵual velocidad, con una caída de presión a expensas de la viscosidad (fricción viscosa) que se opone a dicho movimiento (flujo laminar). La capa más externa (pegada a la pared del tubo), debido a la elevada fricción tiene una velocidad muy pequeña, aumentando en las capas centrales, es decir que su superficie externa en contacto con capas cada vez más periféricas, es mayor, siendo por lo tanto cada vez mayor la resistencia viscosa, a igual presión ("empuje") aplicada a cada una de las capas del flujo laminar. Esta fuerza es opuesta a la circulación del aire produce un retardo sucesivo desde el centro hacia la periferia del tubo, lo que puede ser considerado también como la transferencia del "momento" (o fuerza tangencial al eje de circulación) entre las distintas capas de fluido concéntricas hacia la pared del tubo $(29,32)$.

Recientemente nosotros hemos comunicado que en deportistas existe un aumento lineal de la distancia de seguridad con respecto al flujo respiratorio (VE), lo que garantizaría el distanciamiento óptimo (17). Sin embargó, en los músicos este hecho consideramos que puede ser irrelevante por varios motivos. Por una parte, existe un gran control en el flujo espiratorio, siendo mucho menor que en los sujetos normales (Tabla II), por otra los músicos no ejercen una fuerza mayor que los controles ni en el FEV1 ni en el PEF. A ello hay que añadir que la frecuencia respiratoria no aumenta, con lo cual tampoco se incrementa la velocidad de salida.

Así mismo, debemos considerar el comportamiento de los flujos, puesto que hay una mezcla de flujo laminar y turbulento $(11,32,33)$. Cuando se incrementa la velocidad del flujo comienzan a aparecer fluctuaciones en el aire, de manera que algunas partículas escapan de la línea y van a parar a otros puntos. Ello implica que la velocidad tiene otras componentes perpendiculares al flujo que hacen que algunas de las partículas se desplacen de la línea. Esto las sitúa en la zona de transición de régimen laminar a turbulento. Si la velocidad sigue aumentando, las inestabilidades son más frecuentes y de mayor intensidad, por lo que un gran número de partículas se desplazan a otras "capas". Todo el aire se ha difuminado por el conducto. Este desorden que hace que las partículas se "cambien de carril" es debido a que existen valores instantáneos de la velocidad no nulos que fluctúan alrededor de 0 en las direceiones radial y circunferencial (son valores positivos y negativos con media temporal nula, ya que no hay flujo neto a través de las paredes de los tubos. También en la componente de la velocidad en la dirección principal del flujo existen fluctuaciones, que hacen que el valor de la velocidad instantánea en un punto no se mantenga constante, a pesar de estar en régimen permanente $(11,32,33)$. Pero, además, debemos tener en cuenta el efecto de la divergencia de los fluidos aéreos a través de los tubos (de los instrumentos musicales), lo que hace perder presión cuando el aire es expulsado.

En las ramificaciones (las llaves del instrumento musical), el área de cada una de las ramas hijas (las llaves) es menor que la sección originaria y la suma de las secciones de las ramas hijas es mayor que la de la principal. Debido a que el flujo debe ser constante para obtener un buen sonido y una entonación adecuada, debe existir una caída de la velocidad proporcional en cada una de las ramificaciones estabilizando el flujo.

Aplicando ecuación de Bernoulli $(32,33)$ se deberían aplicar los valores promedio de la velocidad en cada sección del instrumento musical. El cálculo de la velocidad media de una sección es sencillo, y más aún en el caso de un flujo laminar. Sin embargo, utilizar directamente tal valor de velocidad media sobre la ecuación de Bernoulli sería incorrecto, pues la velocidad aparece elevada al cuadrado. La solución para poder hacerlo pasa por utilizar el denominado co- 
eficiente corrector de la energía cinética, es decir, del cálculo del flujo de energía cinétiea por unidad de tiempo que atraviesa una sección. Sin embargo, en la realidad también deberemos tener en cuenta el efecto de atrapamiento del material genético (la proteína del SARS-CoV-2) que el músico infectado espira, pero que se queda dentro del instrumento.

Por lo tanto, se produce una dispersión de la presión del flujo, dada la pérdida que se produce al salir el aire por los distintos orificios, haciendo que la dispersión de las gotitas contenidas en el flujo aéreo tenga menos recorrido que cuando respiramos directamente.

A ello deberíamos añadir, que gran parte de la condensación del aire respirado y espirado por los músicos, se deposita en forma de gotas grandes que caen perpendicularmente a la campana de los instrumentos. No es infrecuente ver, sobre todo a los trompetistas como de vez en cuando tienen que vaciar (sacudir) la acumulación de agua en el instrumento.

Una limitación del estudio es el escaso número de músicos, pero debemos considerar que ellos forman parte de un grupo poblacional de la provincia más pequeña de España (Soria) y por lo tanto el número de músicos es escaso. Y, sin embargo, nuestra provincia ha sido la de mayor incidencia proporcional de coronavirus de toda España.

En conclusión, creemos que en el caso de los músicos debería ser reconsiderada la distancia de seguridad establecida actualmente como prevención de contagio de COVID-19, puesto que los flujos espiratorios son menores y los músicos no están en frente uno de otro (están detrás), además de que el público se encuentra a una distancia de unos 5 metros. Además, deberíamos contemplar que el hecho de la distancia de los tubos hace que al final, la salida de aire sea menor pues parte se queda en el instrumento (espacio muerto). Es decir, en el músico, además de ejercer menor presión para el flujo respiratorio, se controla mucho más acorde también a lo que representa el PIP, y a lo que supone el espacio muerto anatómico del propio instrumento musical. Por otra parte, la condensación del aire exhalado deja mucha cantidad del aerosol en el propio instrumento en forma de gotas que los músicos deben limpiar del instrumento, que en esas condiciones conlleva la higienización del instrumento. Así pues, hay numerosas variables que influyen en los flujos y la dispersión, la propagación de bioaerosoles que deberían ser consideradas.

\section{AGRADECIMIENTOS}

Agradecemos la participación voluntaria y desinteresada de los componentes de la banda municipal de Soria, por su participación e implicación en el estudio.

\section{REFERENCIAS}

1. Cordova A. Fisiología Dinámica 2003. Barcelona: Masson.

2. García-Río F, Calle M, Burgoos F, Casan P, Del Campo F, Galdiz JB, Giner J, González-Mangado N, Ortega F, Puente L, SEPAR. Spirometry. Spanish Society of Pulmonoloǵy and Thoracic Surǵery (SEPAR). Arch Bronconeumol 2013;49:388-401. https:// doi: 10.1016/j.arbres.2013.04.001.

3. Kampf G, Todt D, Pfaender S, Steinmann E. Persistence of coronaviruses on inanimate surfaces and their inactivation with biocidal aǵents. J Hosp Infect 2020;104:246-251. https:// doi: 10.1016/j.jhin.2020.01.022

4. Organización Mundial de la Salud. Vías de transmisión del virus de la COVID-19: repercusiones para las recomendaciones relativas a las precauciones en materia de prevención y control de las infecciones. Ginebra: Orǵanización Mundial de la Salud, 2020. https://www.who.int/es/news-room/ commentaries/detail/modes-of-transmission-of-virus-causing-covid-19-implicationsfor-ipc-precaution-recommendations.

5. Bourouiba L, Dehandschoewercker E, Bush JWM. Violent expiratory events: on coughing and sneezing. J Fluid Mech 2014; 745:537-563.

Vol. 62(1): 16 - 27, 2021 
6. Bourouiba L. Turbulent gas clouds and respiratory pathogen emissions potential implications for reducing transmission of COVID-19. JAMA, 2020; March26. https:// doi: 10.1001/jama.2020.4756.

7. Yu ITS, Li Y, Wong TW, Tam W, Chan AT, Lee JH, Leung DY, Ho T. Evidence of airborne transmission of the severe acute respiratory syndrome virus. N Eng1 J Med 2004; 350: 1731-1739. https://doi: 10.1056/NEJMoa032867.

8. Bahl P, Doolan C, de Silva C, Chughtai AA, Bourouiba L, MacIntyre CR. Airborne or droplet precautions for health workers treating COVID-19? J Infect Dis 2020; 16; jiaa189. https://doi: 10.1093/infdis/ jiaa189

9. Cui Y, Zhang ZF, Zhao F, Wang H, ShunZhang Y, Detels R. Air pollution and case fatality of SARS in the People's Republic of China: an ecologic study. Environ Health 2003; 2(1) :15. https://doi: 10.1186/1476069X-2-15.

10. Loudon RG, Roberts RM. Droplet expulsion from the respiratory tract. Am Rev Respir Dis 1967; 95:435-442. doi: 10.1164/ arrd.1967.95.3.435.

11. Mittal R, Ni R, Seo J. The flow physies of COVID-19. J Fluid Mech 2020:894, F2, doi:10.1017/ifm.2020.330.

12. Morawska L, Cao J. Airborne transmission of SARS-CoV-2: the world should face the reality. Environ Int 2020; 10:105730. https://doi: 10.1016/j.envint.2020.105730

13. Puente L, Marín JM, Burgos F, Cobos N, Casanova C, Cueto A. SEPAR 2011: Procedimientos de evaluación de la función pulmonar. Ed. Manual SEPAR. Madrid: Luzan; 2011.

14. Sant'Anna CA, Montenegro MR, Calanzo de Aquino C,i Klouczek MV. Análise da função pulmonar em músicos que tocam instrumento de sopro. O Mundo da Saúde Sao Paulo 2010; 34:200-209. http://www.saocamilo-sp.br/pdf/mundo_ saude/75/200a209.pdf

15. Fuhrmann AG, Franklin PJ, Hall GL. Prolonged use of wind or brass instruments does not alter lung function in musicians. Respir Med 2011; 105:761-767. https:// doi: 10.1016/j.rmed.2011.01.017
16. Brzęk A, Famuła A, Kowalezyk A, Plinta R. Efficiency of lung ventilation for people performing wind instruments. Med $\mathrm{Pr}$ 2016; 67:427-433. https:// doi: 10.13075/ mp.5893.00398.

17. Córdova A, Latasa I. Respiratory flows as a method for safely preventing the coronavirus transmission (Covid-19). Apunts Sports Med 2020; 55:81-85. https://doi. org/10.1016/j.apunsm.2020.05.001.

18. Official Statement of the European Respiratory Society. Standardized Lung Function Testing. Update 1993. Eur Respir J 1993; 6 (Suppl):1-100.

19. Rohwer D. Adult musicians' perceived and measured pulmonary function. Med Probl Perform Art 2009; 24:10-14.

20. Hajda T. [School for clarinet]. [Kraków: Polskie Wydawnictwo Muzyezne; Polish]. 2013.

21. Borgia JF, Horvath SM, Dunn FR, von Phul PV, Nizet PM. Some physiological observations on French horn musicians. J Occup Med 1975;17: 696-701.

22. Navratil M, Rejsek K. Lung function in wind instrument players and glassblowers. Ann NY Acad Sci 1968; 155:276-282. https://doi. org/10.1111/j.1749-6632.1968.tb56772.x

23. Zuskin E, Mustajbegowic J, Schachter EN, Kern J, Vitale K, Pucarin-Cvetkovic J, Chiarelli A, Milosevic M, Jelinic JD. Respiratory function in wind instrument players. Med Lav 2009; 100:133-141.

24. Harris LR. Horn playing and blood pressure. Lancet 1996; 348:1042. https://doi: 10.1016/S0140-6736(05)64982-3

25. Aguilar J, Carreras A, Teixido A, Haro M, Rodenstein DO, Morera J. Maximum respiratory pressures in trumpet players. Chest 1993; 104:1203-1204. https://doi: 10.1378/chest.104.4.1203.

26. Cossette I, Sliwinski P, Macklem PT. Respiratory parameters during professional flute playing. Respir Physiol 2000; 121:33-44. https://doi:10.1016/s0034-56 87(00)00111-0.

27. Fuhrmann AG, Franklin PJ, Hall GL. Prolonged use of wind or brass instruments does not alter lung function in musicians. Respir Med 2011; 105:761-767. https://doi: 10.1016/j.rmed.2011.01.017. 
28. Large J. Observation on the vital capacity of singers. JASA 1972; 52, 147. https://doi. org $/ 10.1121 / 1.1981925$.

29. OIT (Organización Internacional del Trabajo). Las normas de la OIT y la COVID-19 (coronavirus) https://www.ilo.org/wemsp5/groups/public/---ed_norm/---normes/ documents/publication/wems_739939.pdf

30. Stauffer DW. Physical performance, selection and training of wind instrument players. Ann NY Acad Sci. 1968; 155:284-289. https:// doi: 10.1111/j.1749-6632.1968.tb5 6773.x
31. Li Y, Leung GM, Tang JW, Yang $X$, Chao CY, Lin JZ, Lu JW, Nielsen PV, Niu J, Qian H, Sleigh AC, Su HJ, Sundell J, Wong TW, Yuen PL. Role of ventilation in airborne transmission of infectious agents in the built environment. A multidisciplinary systematic review. Indoor Air 2007; 17: 2-18.

32. Mott RL. Mecánica de fluidos. México. Pearson Educación, 2006.

33. 33. Arregui FJ, Cabrera E, Cobacho R, Gómez E, Soriano J. Apuntes de mecánica de fluidos. Valencia. Universidad Politécnica, 2017. 